\title{
Make MicroED an efficient tool for ultrahigh-resolution structural determination?
}

\author{
$X \mathrm{Li}^{1}$ \\ ${ }^{1}$ Tsinghua University, Beijing \\ lixueming@tsinghua.edu.cn
}

Microcrystal electron diffraction (MicroED) is becoming a powerful tool in determining the crystal structures of biological macromolecules and small organic compounds. An advantage of electron diffraction is that its resolution is just weakly influenced by the performance of the objective lens. Therefore, even on a low-end electron microscope the electron diffraction can reach half angstrom resolution. By developing a stage-camera synchronization scheme to minimize the hardware requirements and enable the use of the conventional electron cryo-microscope with single-frame CCD camera, the acquisition of ultrahigh-resolution diffraction data is achieved on conventional microscopes, including the low-end $120 \mathrm{kV}$ microscopes. This method was demonstrated by the structure determination of both peptide and small organic compounds at ultrahigh resolution up to $\sim 0.60$ angstrom with unambiguous assignment of nearly all hydrogen atoms. Importantly, this work also demonstrates the capability of the low-end $120 \mathrm{kV}$ microscope with a CCD camera in solving ultra-high resolution structures of both organic compound and biological macromolecules. To develop a routinely-available method for high-quality MicroED sample, we used the focused ion beam (FIB) equipped on the scanning electron microscope (SEM) to mill a large crystal to thin lamella. The influences of the milling on the crystal lamella were observed and investigated, and used to optimize the FIB milling. FIB also makes MicroED available for crystals with large size.

Acta Cryst. (2020). A76, a203 